HU-SEFT R 1995-09

CBPF-NF-022/95

\title{
Path Integrals with Generalized Grassmann Variables
}

\author{
M. Chaichian \\ High Energy Physics Laboratory, Department of Physics \\ and Research Institute for High Energy Physics, \\ University of Helsinki \\ P.O.Box 9 (Siltavuorenpenger 20 C), FIN-00014, \\ Helsinki, Finland \\ and \\ A.P.Demichev日 \\ Centro Brasileiro de Pesquisas Fisicas - CBPF/CNPq, \\ Rua Dr.Xavier Sigaund, 150, \\ 22290-180, Rio de Janeiro, \\ RJ Brasil
}

\begin{abstract}
We construct path integral representations for the evolution operator of q-oscillators with root of unity values of q-parameter using BargmannFock representations with commuting and non-commuting variables, the differential calculi being q-deformed in both cases. For $q^{2}=-1$ we obtain a new form of Grassmann-like path integral.
\end{abstract}

\footnotetext{
${ }^{\dagger}$ on leave of absence from Nuclear Physics Institute, Moscow State University, 119899, Moscow, Russia
} 


\section{Introduction}

Path integrals over Grassmann variables play important role in modern field theory. In the simplest case of one degree of freedom it describes the quantum mechanical system with two possible states. It is natural to generalize the consideration for the case of quantum mechanical system with arbitrary but finite number of states. As is well known, operator description of such systems is realized with help of q-oscillators algebra [1], parameter $q$ being root of unity: $q^{p}=1$ for some integer $p$. Note that in general quantum algebras in the root of unity case exhibit rich representation behaviours with very special properties (including bound on their dimensionality) and are important in two-dimensional conformal field theories [2] and in statistical mechanics models [3]. We shall consider even integer $p$, so that $q^{(k+1)}=-1$ for $k=p / 2-1$. The case $q^{2}=-1$ corresponds to the usual fermionic oscillator. Of course, generalization to field theory requires the consideration of more complicated algebra of system of q-oscillators and its precise physical meaning depends on the chosen form of commutation relations for the different q-oscillators (bosonic excitations on lattice with finite number of states at a given site or system with fractional statistics). Here we consider one degree of freedom only and the aim of this letter is to present possible Bargmann-Fock representations of the q-oscillators with finite Fock space and to construct corresponding q-deformed path integral. An interesting attempt to acheive this was made in [4] but as we discussed earlier [5] the authors did not take into account that the creation and destruction operators used by them are not conjugated to each other in the root of unity case and as the final result they obtained discrete approximation for the path integral representation of (non-unitary) evolution operator.

If the Hilbert space of a system corresponds to representation of some Lie group the corresponding path integral can be constructed with help of generalized coherent states (see, e.g., [6] and refs. therein). For our construction it is not necessary to assume that there is any symmetry. In general we shall follow the method suggested by us in [5] for the q-deformed path integral in the case of real q-parameter. It is interesting that even in the simplest Grassmann-like case $q^{2}=-1$ there are different forms of BF representation and path integrals depends on the way how the Planck constant $\hbar$ enters the commutation relation. To generalize the usual Grassmann path integral for the case of higher roots we suggest another algebra of variables on different time slices, the Grassmann integral being independent of the modification.

Such path integrals can be useful for consideration of systems on a lattice [7], in anyon physics [8], for description of quantum particles on finite sets of points [9] and for q-deformed string theory [10]. 


\section{Path integrals for different choices of variables}

The usual starting commutation relations (CR) for q-oscillator operators is [1]

$$
a a^{\dagger}-q a^{\dagger} a=q^{-N}, \quad a^{\dagger} a=[N]_{q} \equiv \frac{q^{N}-q^{-N}}{q-q^{-1}},
$$

where $q^{k+1}=-1$ for some integer $k$ and $a^{\dagger}$ is hermitian conjugated to $a$. To construct the Bargmann-Fock (BF) representation we introduce the operators $b$ and $\bar{b}$ (well known in the case of real q-parameter)

$$
b=q^{N / 2} a, \quad \bar{b}=a^{\dagger} q^{N / 2}
$$

with CR

$$
\begin{gathered}
b \bar{b}-q^{2} \bar{b} b=1, \\
b \bar{b}-\bar{b} b=q^{2 N} \\
\bar{b} b=[N] \equiv \frac{q^{2 N}-1}{q^{2}-1} .
\end{gathered}
$$

For root of unity value of parameter $q$ operator $\bar{b}$ is not hermitian conjugate to $b$, but instead

$$
b^{\dagger}=\bar{b} q^{-N}, \quad \bar{b}^{\dagger}=q^{-N} b .
$$

Fock space representation of the operators is the following

$$
b|n\rangle=q^{n-1} \sqrt{[n]_{q}}|n-1\rangle, \quad \bar{b}|n\rangle=\sqrt{[n+1]_{q}}|n+1\rangle, \quad N|n\rangle=n|n\rangle .
$$

As $[k+1]_{q}=0$, this Fock space is k-dimensional.

There are two possibilities for explicit realization of (3). One can construct BF representation in the space of antiholomorphic functions with the basis

$$
\psi_{n}=\frac{\bar{z}^{n}}{\sqrt{[n]_{q} !}},
$$

with either commuting $\bar{z}, z$ variables or non-commuting ones. In both cases the variables must satisfy the condition of nilpotence $z^{k+1}=\bar{z}^{k+1}=0$ to provide the finiteness of the Fock space.

We shall consider this on the example of Grassmann-like $q^{2}=-1$ case 1 . Physicaly the possibilities correspond to the different forms of the CR (1),(2) after recovering of Planck constant $\hbar$. For $q^{2}=-1$ case and in representation (3) both CR (11) and (2) become the same

$$
b \bar{b}+\bar{b} b=1
$$

\footnotetext{
${ }^{1}$ We call this case Grassmann-like because though operators $b$ and $\bar{b}$ satisfy the anticommutation relations, we use for the construction of the path integrals either commuting variables or variables with non-Grassmann algebra on different time slices.
} 
As usual one understands that Planck constant in this CR is equal to unity. But it is possible to recover it in two different ways. One way is to present (4) in the form

$$
[b, \bar{b}]=\hbar(1-2 \gamma \bar{b} b)
$$

where $\gamma$ is dimensionful constant such that $\hbar \gamma=1$. This CR corresponds to a curved phase space dynamics with Poisson braket

$$
\{z, \bar{z}\}_{P}=i(1-2 \gamma \bar{z} z) \equiv i \omega^{-1}(\bar{z} z)
$$

where $z, \bar{z}$ are the classical counterparts of $b, \bar{b}$ and $\omega=\omega(\bar{z} z) d z \wedge d \bar{z}$ is the symplectic form. Evolution operator corresponding to such quantization must be expressed with help of q-path integral with commuting variables.

The key observation for the developing of path integral representations is that $\mathrm{CR}$ written in the form (1) does not lead unavoidably to $\mathrm{BF}$ variables with $\mathrm{CR} z \bar{z}=q^{2} \bar{z} z$, but to CR

$$
\bar{\partial} \bar{z}-q^{2} \bar{z} \bar{\partial}=1
$$

only. So one can consider two parametric differential calculus on the quantum plane 11] and for the case of curved phase space (5) choose the commuting complex variables $z \bar{z}=\bar{z} z$ with the following nontrivial CR only

$$
\bar{\partial} \bar{z}+\bar{z} \bar{\partial}=1, \quad d \bar{z} \bar{\partial}=-\bar{\partial} d \bar{z}, \quad \bar{z} d \bar{z}=-(d \bar{z}) \bar{z}
$$

with their conjugated counterparts. The creation $\bar{b}=\bar{z}$ and destruction $b=\bar{\partial}$ operators act in the BF space with the basis $\left\{\psi_{0}=1, \psi_{1}=\bar{z}\right\}$ and the scalar product

$$
\int d \bar{z} d z e^{\bar{z} z} \bar{\psi}_{n} \psi_{m}=\delta_{n m}
$$

where the integral is defined by the usual Berezin rules

$$
\int d \bar{z} \bar{z}=\int d z z=1, \quad \int d \bar{z}=\int d z=0 .
$$

The method of the path integral construction is analogous to that in the case of usual Grassmann path integral (see e.g. [12]). As a result the evolution operator kernel takes the form

$U\left(t^{\prime \prime}-t^{\prime}\right)=\int\left(\prod_{t} \frac{d \bar{z}(t) d z(t)}{1-2 \bar{z}(t) z(t)}\right) \exp \left\{\bar{z}\left(t^{\prime \prime}\right) z\left(t^{\prime \prime}\right)-\int_{t^{\prime}}^{t^{\prime \prime}}(\bar{z}(t) \dot{z}(t)+i H(\bar{z}(t) z(t))) d t\right\}$

with $\dot{z}(t)$ denoting the usual derivative with respect to time: $\dot{z}(t)=d z(t) / d t$. Note that the integral measure corresponds to the form of nontrivial Poisson bracket (6) after putting $\gamma=\hbar=1$.

If after the recovering of Planck constant the CR (四) takes the form

$$
b \bar{b}+\bar{b} b=\hbar
$$


then it corresponds to Grassmann phase space with anticommuting variables. In this case the BF variables seems to be identical to the usual Grassmann path integral. But there is one subtlety. In the usual construction not only $z\left(t_{i}\right)$ and $\bar{z}\left(t_{j}\right)$ anticommute for any time slices $t_{i}, t_{j}$ but the same variables on different time slices anticommute also, e.g. $z\left(t_{i}\right) z\left(t_{j}\right)+z\left(t_{j}\right) z\left(t_{i}\right)=0$. Such CR can not be generalized to other roots of unity values of parameter $q$. So we introduce another CR on different time slices (cf. [5])

$$
\begin{array}{cc}
z\left(t_{i}\right) \bar{z}\left(t_{j}\right)+\bar{z}\left(t_{j}\right) z\left(t_{i}\right)=0, & z^{2}\left(t_{i}\right)=\bar{z}^{2}\left(t_{i}\right)=0, \\
\bar{z}\left(t_{i}\right) \bar{z}\left(t_{j}\right)=\bar{z}\left(t_{j}\right) \bar{z}\left(t_{i}\right), & z\left(t_{i}\right) z\left(t_{j}\right)=z\left(t_{j}\right) z\left(t_{i}\right) .
\end{array}
$$

One can check that for such CR all ingredients of path integral construction (scalar product measure, relation between normal symbol and kernel of operators, etc.) remain the same as in the case of usual Grassmann path integral. So the result proved to be the same also.

The CR (9) can be easily generalized to other values of deformation parameter, for example, for $q^{3}=-1$ one has

$$
\begin{array}{cc}
z\left(t_{i}\right) \bar{z}\left(t_{j}\right)=q^{2} \bar{z}\left(t_{j}\right) z\left(t_{i}\right), & z^{3}\left(t_{i}\right)=\bar{z}^{3}\left(t_{i}\right)=0 \\
\bar{z}\left(t_{i}\right) \bar{z}\left(t_{j}\right)=\bar{z}\left(t_{j}\right) \bar{z}\left(t_{i}\right), & z\left(t_{i}\right) z\left(t_{j}\right)=z\left(t_{j}\right) z\left(t_{i}\right) .
\end{array}
$$

In this case the BF representation is defined on the space of function with the basis

$$
\psi_{0}=1, \quad \psi_{1}=\bar{z}, \quad \psi_{2}=\bar{z}^{2},
$$

which is orthonormal with respect to the scalar product

$$
\int d \bar{z} d z \overline{\psi_{n}(\bar{z})} \mu(\bar{z} z) \psi_{m}(\bar{z})=\delta_{n m}
$$

where

$$
\mu(\bar{z} z)=1+q^{2} \bar{z} z+q^{2} \bar{z}^{2} z^{2} .
$$

Here the integral is defined by the relation 4 :

$$
\int d \bar{z} d z z^{n} \bar{z}^{m}=\delta_{n k} \delta_{m k}[k]_{q} !, \quad n, m=0,1,2 .
$$

Because of the nilpotence a general Hamiltonian has the form

$$
H=\omega\left(u \bar{b} b+v \bar{b}^{2} b^{2}\right)
$$

where the constants $u, v$ are restricted by the hermiticity condition $H^{\dagger}=H$ and can take three couples of the values: $i) u=1, v=-q$; ii) $u=1, v=1-2 q$; iii) $u=0, v=-q^{2}$. In this case and in the cases of higher roots of unity the measure of scalar product in BF spaces and relation between normal symbols and kernels of operators are not expressed with help of appropriate q-deformed exponents. This prevents from writing the general expression of path integral for arbitrary root of 
unity. But due to nilpotence for any given value of parameter $q$ it can be done analogously to the case of real q-parameter [5].

As usual the action of any operator $A$ in BF Hilbert space can be represented with the help of its kernel $\mathcal{A}$

$$
(A f)\left(\bar{z}_{1}\right)=\int d \bar{z}_{2} d z_{2} \mathcal{A}\left(\bar{z}_{1}, z_{2}\right) f\left(\bar{z}_{2}\right)
$$

where

$$
\mathcal{A}\left(\bar{z}_{1}, z_{2}\right)=\sum_{m, n=0}^{2} A_{m n} \bar{z}_{1}^{m} z_{2}^{n} .
$$

Here one more pair of q-commuting coordinates is introduced, the CR for different pairs being defined by (10). Now we can express $A_{m n}$ through scalar product

$$
A_{m n}=<\psi_{m}\|A\| \psi_{n}>
$$

and find a kernel of any operator by direct calculation. We consider the usual Schrödinger equation (cf. discussion in [5])

$$
i \frac{d}{d t} \Psi(\bar{z}, t)=H(\bar{b}, b) \Psi(\bar{z}, t)
$$

with the Hamiltonian (14). The integral kernel for the infinitesimal operator

$$
U \approx 1-i H \Delta t
$$

takes the form

$$
U(\bar{z}, z ; \Delta t) \approx g(\bar{z} z) \exp \left\{-i H_{e f f} \Delta t\right\},
$$

where $g(\bar{z} z)$ is the kernel of identity operator

$$
g(\bar{z} z)=\sum_{n} \psi_{n}(\bar{z}) \overline{\psi_{n}(\bar{z})}=1+\bar{z} z+\bar{z}^{2} z^{2}
$$

and effective Hamiltonian $H_{\text {eff }}$ is defined by the relation

$$
H_{e f f}(\bar{z} z)=g^{-1}(\bar{z} z) \mathcal{H}(\bar{z} z),
$$

where $\mathcal{H}(\bar{z} z)$ is kernel of the initial Hamiltonian. Using the nilpotence one obtains from (20)

$$
H_{e f f}(\bar{z} z)=\omega\left(u \bar{z} z+q(u+v-q u) \bar{z}^{2} z^{2}\right) .
$$

Now we can write the convolution of $K$ infinitesimal evolution operator kernels

$$
\begin{aligned}
& U\left(\bar{z}_{K} z_{K-1}\right) * U\left(\bar{z}_{K-1} z_{K-2}\right) * \ldots * U\left(\bar{z}_{1} z_{0}\right)= \\
= & \int d \bar{z}_{K-1} d z_{K-1} \ldots d \bar{z}_{1} d z_{1} \mu\left(\bar{z}_{K-1} z_{K-1}\right) \ldots \mu\left(\bar{z}_{1} z_{1}\right)
\end{aligned}
$$




$$
\times g\left(\bar{z}_{K} z_{K-1}\right) \ldots g\left(\bar{z}_{1} z_{0}\right) e^{-i H_{e f f}\left(\bar{z}_{K} z_{K-1}\right) \Delta t} \ldots e^{-i H_{e f f}\left(\bar{z}_{1} z_{0}\right) \Delta t} .
$$

Due to nilpotence it is possible to convert the functions $g$ and $\mu$ to the exponents and in the continuous limit $\Delta t \rightarrow 0$ one obtains the path integral for the case under consideration $\left(q^{3}=-1\right)$

$$
\begin{aligned}
U\left(t^{\prime \prime}-t^{\prime}\right)= & \int\left(\prod_{t} d \bar{z}(t) d z(t)(1+q \bar{z}(t) z(t))\right)\left(1+\bar{z}\left(t^{\prime \prime}\right) z\left(t^{\prime \prime}\right)+\bar{z}^{2}\left(t^{\prime \prime}\right) z^{2}\left(t^{\prime \prime}\right)\right) \\
& \times \exp \left\{-\int_{t^{\prime}}^{t^{\prime \prime}}\left[(1+(1+2 q) \bar{z} z) \bar{z}(t) \dot{z}(t)+i H_{e f f}(\bar{z}(t) z(t))\right] d t\right\} .
\end{aligned}
$$

The path integrals for higher roots can be derived in the same way. For example, for the next value $q^{4}=-1$ one has

$$
\begin{array}{r}
U\left(t^{\prime \prime}-t^{\prime}\right)=\int\left(\prod _ { t } d \overline { z } ( t ) d z ( t ) \left[1+(1+i) \bar{z}(t) z(t)+(2 c-1) \bar{z}^{2}(t) z^{2}(t)+\right.\right. \\
\left.\left.i(1-c) \bar{z}^{3}(t) z^{3}(t)\right]\right) \exp \left\{\bar{z}\left(t^{\prime \prime}\right) z\left(t^{\prime \prime}\right)+(c-i / 2) \bar{z}^{2}\left(t^{\prime \prime}\right) z^{2}\left(t^{\prime \prime}\right)+(2 c-i / 3) \bar{z}^{3}\left(t^{\prime \prime}\right) z^{3}\left(t^{\prime \prime}\right)\right\} \\
\times \exp \left\{-\int_{t^{\prime}}^{t^{\prime \prime}}\left[\left(1-(1 / 2+i c) \bar{z} z-3(2 c-i / 2) \bar{z}^{2} z^{2}\right) \bar{z}(t) \dot{z}(t)+i H_{e f f}(\bar{z}(t) z(t))\right] d t\right\},
\end{array}
$$

where $c \equiv 2^{-1 / 4}=1 / \sqrt{[2]_{q}}$ and $H_{\text {eff }}$ is defined again by the relation (20) with the corresponding kernel

$$
g(\bar{z} z)=1+\bar{z} z+c \bar{z}^{2} z^{2}+c \bar{z}^{3} z^{3} .
$$

The kernels $U$ in Eqs. (22) and (23) correspond to the product of unitary up to a $(\Delta t)^{2}$-terms operators with subsequent limit $\Delta t \rightarrow 0$. So these kernels seem to be the kernels of unitary evolution operators. However, the integrands of these path integrals have not the form $\exp \{i S\}$, where $S$ is a real functional. This is quite unusual and prevents from ordinary interpretation of a path integral. The obvious reason for this is the CR (10) for $z$ and $\bar{z}$ coordinates which contain complex parameter $q$.

Let us consider for $q^{3}=-1$ another possibility using commuting variables with CR (7) for variables and derivatives in analogy with Grassmann-like case. The basis of BF representation has the same form (11) as in the case of noncommuting variables and is orthonormal with respect to scalar product (12) with the mesure $\mu=1+\bar{z} z+\bar{z}^{2} z^{2}$, the integral being defined by (13) (but now with commuting variables). The derivation of the path integral is essentially the same as in the case of noncommuting variables and the result is

$$
\begin{array}{r}
U\left(t^{\prime \prime}-t^{\prime}\right)=\int\left(\prod_{t} d \bar{z}(t) d z(t)\left(1+2 \bar{z}(t) z(t)+3 \bar{z}^{2}(t) z^{2}(t)\right)\right)\left(1+\bar{z}\left(t^{\prime \prime}\right) z\left(t^{\prime \prime}\right)\right. \\
\left.+\bar{z}^{2}\left(t^{\prime \prime}\right) z^{2}\left(t^{\prime \prime}\right)\right) \exp \left\{-\int_{t^{\prime}}^{t^{\prime \prime}}\left[(1+\bar{z} z) \bar{z}(t) \dot{z}(t)+i H_{\text {eff }}(\bar{z}(t) z(t))\right] d t\right\} .
\end{array}
$$


The effective Hamiltonian is defined by the general formula (20) again, but now for all hermitian Hamiltonians of the form (14) listed above $H_{\text {eff }}$ proved to be real function: i) $H_{e f f}=\omega \bar{z} z$ for $u=1, v=-q$; ii) $H_{\text {eff }}=\omega\left(\bar{z} z-\bar{z}^{2} z^{2}\right)$ for $u=1, v=1-2 q$; iii) $H_{\text {eff }}=\omega \bar{z}^{2} z^{2}$ for $u=0, v=-q^{2}$. Note that the normal symbols and the kernels of all hermitian Hamiltonians are complex. Thus in the case of commuting variables $\bar{z}$ and $z$ the path integral has the usual form $\exp \{i S\}$ of integrand with real functional $S$ even for complex parameter $q$ of an oscillator deformation.

After restoring the Planck constant $\hbar$ the expression (24) takes the form (for definiteness we consider the Hamiltonian (20) with $u=1, v=-q$ )

$$
\begin{aligned}
U\left(t^{\prime \prime}-t^{\prime}\right) & =\int\left[\prod_{t} \frac{d \bar{z}(t) d z(t)}{\hbar}\left(1+2 \frac{\bar{z}(t) z(t)}{\hbar}+3 \frac{\bar{z}^{2}(t) z^{2}(t)}{\hbar^{2}}\right)\right]\left(1+\frac{\bar{z}\left(t^{\prime \prime}\right) z\left(t^{\prime \prime}\right)}{\hbar}\right. \\
& \left.+\frac{\bar{z}^{2}\left(t^{\prime \prime}\right) z^{2}\left(t^{\prime \prime}\right)}{\hbar^{2}}\right) \exp \left\{-\frac{1}{\hbar} \int_{t^{\prime}}^{t^{\prime \prime}}\left[\left(1+\frac{\bar{z} z}{\hbar}\right) \bar{z}(t) \dot{z}(t)+i \omega \bar{z}(t) z(t)\right] d t\right\} .
\end{aligned}
$$

One can see that there is simply no quasiclassical approximation corresponding to the limit $\hbar \rightarrow 0$ (since the terms in the measure and in the action diverge). This is an expected result because, as it is well known, the quasiclassical approximation for spin-like systems considered here corresponds to the limit $S \rightarrow \infty$, where $\mathrm{S}$ is the spin of the system [13]. Systems with fixed spins (numbers of states) have not (quasi)classical limit, as is confirmed once again by the expression (25).

Let us notice that while the real phase space variables (coordintaes and momenta) are not suitable in describing spin systems, the BF basis is. The simplest and well known example is the Grassmann algebra variables for describing spin $1 / 2$ particles. It is also of interest to mention that in a recent attempt to formulate a regularized quantum field theory by requiring nonzero minimal uncertainties in the positions and in the momenta, for the real q case the use of Bargmann-Fock space appears to be the appropriate one [14. The q-deformed path integral previously given [5] for the real q values, has also been formulated in the BF basis emerged in a natural way.

The fact that creation and destruction operators $\bar{b}, b$ are not hermitian conjugate is quite unusual and technically inconvenient (but as they are non-conjugate up to pure phase only this does not cause real problems). One can try to use hermitian conjugated operators $a^{\dagger}, a$ for the path integral construction noticing that the relation (1) is in fact a polynomial one due to nilpotence. In particular, in $q^{3}=-1$ case and in the Fock space representation the relation (1) is equivalent to the CR

$$
a a^{\dagger}+\left(a^{\dagger}\right)^{2} a^{2}=1
$$

This CR looks like a natural generalization of the usual relation (4) for fermionic operators 2 . To construct the BF representation for (26) one must introduce unusual

\footnotetext{
${ }^{2}$ One can show that the algebra of operators $\alpha \alpha^{\dagger}+\left(\alpha^{\dagger}\right)^{n} \alpha^{n}=1$, has $(n+1)$-dimensional Fock representation; a general consideration of polynomial algebras with finite-dimensional Fock representations will be given elsewhere.
} 
"differential" operators $\mathcal{D}, \overline{\mathcal{D}}$ with the properties

$$
\begin{gathered}
\overline{\mathcal{D}} \bar{z}+\bar{z}^{2} \overline{\mathcal{D}}^{2}=1, \quad \mathcal{D} z+z^{2} \mathcal{D}^{2}=1, \\
\bar{z}^{3}=z^{3}=1 .
\end{gathered}
$$

Consider for definiteness the commuting variables $z \bar{z}=\bar{z} z$ (one can consider other possibilities, e.g. anticommuting $z$ and $\bar{z}$ ). The basis of the functions (11) is orthonormal with respect to the scalar product (12) with function $\mu=1+\bar{z} z+\bar{z}^{2} z^{2}$, the integral being again defined by (13). The operators $\bar{z}$ and $\overline{\mathcal{D}}$ are hermitian conjugate with respect to this scalar product. One can check that the normal symbols $\mathcal{A}_{N}(\bar{z} z)$ of operators $A$ and their kernels $\mathcal{A}(\bar{z} z)$ are related with help of the same function $\mu(\bar{z} z)$ that defines the scalar product, the kernel being defined in the usual way

$$
A \psi(\bar{z})=\int d \bar{z}^{\prime} d z^{\prime} \mathcal{A}\left(\bar{z}, z^{\prime}\right) \psi\left(\bar{z}^{\prime}\right)
$$

Thus using the nilpotence and usual procedure for path integral derivation one obtaines

$$
\begin{aligned}
U\left(t^{\prime \prime}-t^{\prime}\right)=\int( & \left.\prod_{t} d \bar{z}(t) d z(t)\left(1+2 \bar{z}(t) z(t)+3 \bar{z}^{2}(t) z^{2}(t)\right)\right) \mu\left(\bar{z}\left(t^{\prime \prime}\right) z\left(t^{\prime \prime}\right)\right) \\
& \times \exp \left\{-\int_{t^{\prime}}^{t^{\prime \prime}}\left[(1+\bar{z} z) \bar{z}(t) \dot{z}(t)+i H_{e f f}(\bar{z}(t) z(t))\right] d t\right\} .
\end{aligned}
$$

Analogous expressions can be derived for the algebras which correspond to Fock spaces of arbitrary dimension.

\section{Conclusion}

We have succeeded in formulating q-deformed path integrals for root of unity values of q-parameter for simplest nontrivial quantum mechanical systems. Using different differential and integral calculi on quantum plane we constructed the q-deformed Bargmann-Fock representations and path integrals for commuting and non-commuting variables. As we have shown different forms of path integrals are possible even for the well known case of anticommuting creation and destruction operators leading to different Grassmann-like path integrals. The existence of non-standard forms of path integral for fermionic operators permited us to generalize it to the case of qoscillators with higher root of unity q-parameter. The essential distinction of the generalized Grassmann path integrals is that they have a non-trivial integral measure and a non-Gaussian integrand.

\section{Acknowledgements}

A.D.'s work was partially supported by the INTAS-93-1630 grant. 


\section{References}

[1] A.Macfarlane, J.Phys., A22 (1989) 4581;

L.Biedenharn, J. Phys., A22 (1989) L873;

M. Chaichian and P. Kulish, Phys. Lett., B 234 (1990) 72.

[2] L.Alvarez-Gaume, C.Gomes and G.Sierra, Nucl.Phys., B319 (1989) 155.

[3] H.J. de Vega, Nucl.Phys., Nucl.Phys., B374 (1992) 692.

[4] L.Baulieu and E.G.Floratos, Phys.Lett., B258 (1991) 171.

[5] M.Chaichian and A.P.Demichev, Phys.Lett., B320 (1994) 273.

[6] A.Inomata, H.Kuratsuji and C.C.Gerry, Path integrals and coherent states of SU(2) and SU(1,1), World Scientific, Singapore, 1992.

[7] P.Kulish and N.Reshetikhin, J.Sov.Math., 23 (1983) 2435.

L.D.Faddeev and L.A.Takhtajan, Lect.Notes in Physics, 246 (1986) 166.

[8] A.Lerda, Anyons: Quantum mechanics of particles with fractional statistics, Springer, Berlin, 1992.

[9] A.Dimakis, F.Muller-Hoissen and T.Striker, J.Phys., A26 (1993) 1927.

[10] M.Chaichian, J.F.Gomes and R.Gonzalez Felipe, Phys.Lett., B341 (1994) 147.

[11] K.Kowalski and J.Rembielinski, J.Math.Phys., 34 (1993) 2153.

[12] F.A.Berezin, Teor. Mat.Fiz., 6 (1971) 194.

L.D.Faddeev and A.A.Slavnov, Gauge fields. Introduction to quantum theory, Reading Mass., Benjamin, 1980.

[13] F.A.Berezin, Comm.Math.Phys., 40 (1975) 153.

[14] A. Kempf, Quantum field theory with nonzero minimal uncertainties in positions and momenta, preprint DAMTP-94-33, 1994. 